\section{The Canadian Journal of Public Health in 2010}

The beginning of a New Year is often a time to reflect on the past and make wishes for the future. It is particularly appropriate to do so this year as we embark on the second century of existence of the Canadian Public Health Association and its Journal! To this end, we published Historical Notes and Invited Commentaries in 2009 to document and analyze our past and contribute to a debate about future public health challenges. We will continue these features in 2010 and gradually add new ones to address important challenges for public health in the next decade.

Our mission is to publish the best scientific manuscripts we can in the field of public and population health and to contribute to the strengthening of intellectual life of this field in Canada and beyond. There are many ways the Journal can accomplish this, however our means are limited. Journal activities depend on a small but highly effective and dedicated staff, many volunteer reviewers and a few volunteer editorial board members. We will create a more functional editorial board in 2010 and expand its membership to tap into the broad Canadian expertise and to stimulate new ideas and strategies to achieve our goals. We will also invite public health leaders to provide directions and advice to the Journal on an International Advisory Board.

We will add associate editors to oversee regular thematic sections on ethics, the history of public health, intervention research, quantitative and qualitative methods and theories, global health and Aboriginal health; to generate submission of manuscripts; and to stimulate lively debates and discussions in these areas. We will still welcome submissions in all fields of public and population health. Identifying thematic priorities, however, allows us to recognize the importance of some sectors (intervention research, global and Aboriginal health) in achieving meaningful improvements in peoples' health, increasing the knowledge base of researchers and practitioners in multidisciplinary approaches to public health problems (quantitative and qualitative methods and theories) and contributing to the growth of our understanding of essential concepts, ideas, principles and values critical to an effective public health system (history and ethics). These priorities will be revisited regularly to ensure that the Journal remains topical and relevant to the field.

Associate editors and board members are currently being recruited. We invite you to send us your suggestions for further improvements to the Journal and to contribute your best and most relevant manuscripts to enhance public health research and practice over the next decade.

On behalf of the CJPH staff and editorial board, our very best wishes to all our readership for a dynamic and successful year!

\section{Gilles Paradis}

Scientific Editor

\section{La Revue canadienne de santé publique en 2010}

Le début d'une nouvelle année est souvent un temps pour réfléchir au passé et exprimer des souhaits pour l'avenir. Il est particulièrement approprié de le faire cette année à l'amorce du deuxième siècle d'existence de l'Association canadienne de santé publique et de sa Revue! Ainsi, nous avons publié des Notes historiques et des Commentaires invités en 2009 afin de documenter et d'analyser notre passé et de favoriser un débat sur les futurs défis en santé publique. Nous garderons ces chroniques en 2010 et nous en ajouterons progressivement de nouvelles pour aborder les grands défis de la santé publique des 10 prochaines années.

Notre mission est de publier les meilleurs manuscrits scientifiques possibles en santé publique et des populations et de contribuer à renforcer la vie intellectuelle dans ce domaine au Canada et à l'étranger. Il y aurait plusieurs façons d'accomplir ceci avec la Revue, mais nos moyens sont limités. Les activités de la Revue dépendent d'une petite équipe très efficace d'employés à plein temps, de nombreux évaluateurs bénévoles et des quelques membres bénévoles du comité de rédaction. Nous voulons créer un comité de rédaction plus fonctionnel en 2010 et élargir son effectif pour puiser dans le vaste savoir-faire canadien et faire naître de nouvelles idées et de nouvelles stratégies pour atteindre nos objectifs. Nous inviterons aussi des responsables de la santé publique à proposer des orientations et à donner leur avis à la Revue par le biais d'un Conseil consultatif international.

Nous ajouterons des rédacteurs adjoints pour coordonner des rubriques sur les aspects éthiques et l'histoire de la santé publique, la recherche d'intervention, les méthodes et les théories quantitatives et qualitatives, la santé mondiale et la santé autochtone. Grâce à eux, nous voulons aussi recevoir davantage de manuscrits et stimuler des débats et des discussions animés sur les thèmes choisis. Nous accepterons encore des soumissions dans tous les domaines de la santé publique et des populations, mais en cernant nos priorités thématiques, nous soulignons l'importance de certains secteurs (recherche d'intervention, santé mondiale et santé autochtone) pour améliorer concrètement la santé des gens, accroître la base de connaissances des chercheurs et des praticiens dans les approches multidisciplinaires aux problèmes de santé publique (méthodes et théories quantitatives et qualitatives) et mieux comprendre les concepts, les idées, les valeurs et les principes essentiels à un système de santé publique efficace (histoire et éthique). Ces priorités seront examinées périodiquement pour nous assurer d'avoir une Revue actuelle et qui conserve toute sa pertinence pour les gens du domaine.

Nous sommes en train de recruter les rédacteurs adjoints et les membres du Conseil consultatif international et du comité de rédaction. Faites-nous part de vos suggestions pour améliorer la Revue et envoyez-nous vos manuscrits les meilleurs et les plus susceptibles d'améliorer la recherche et la pratique en santé publique au cours de la prochaine décennie.

Au nom du personnel et du comité de rédaction de la RCSP, nous souhaitons à tous nos lecteurs une année prospère et dynamique!

Le rédacteur scientifique, Gilles Paradis 